\title{
RELEVANCIA DE LAS NUEVAS TÉCNICAS DE NEUROIMAGEN PARA LA DETECCIÓN DE ANEURISMAS EN LAS PARÁLISIS AISLADAS DEL III NERVIO CRANEAL
}

\section{RELEVANCE OF THE NEW NEUROIMAGING TECHNIQUES FOR DETECTION OF ANEURYSMS IN ISOLATED THIRD NERVE PALSIES}

\author{
GRAELL X ${ }^{1}$, ARRUGA J², MUÑOZ S ${ }^{3}$, SAN-ROMÁN L L ${ }^{4}$, DE-MIQUEL MA ${ }^{5}$
}

Las parálisis aisladas del III nervio craneal (P3N) pueden ser consecutivas a etiologías diversas, como isquemia periférica, inflamaciones, traumatismos, accidentes vasculares cerebrales y procesos compresivos, que incluyen tumores y aneurismas. De todas ellas, la que conlleva una mayor urgencia vital para el paciente es la compresión del nervio motor ocular común causada por un aneurisma de la arteria comunicante posterior (AAcP). A lo largo de su trayecto por el espacio subaracnoideo, el III nervio guarda una relación muy estrecha con dicha arteria, que une la circulación de la arteria cerebral posterior (rama de la arteria basilar) con la de la arteria carótida interna. Precisamente, es en la proximidad de la unión de esta última con la comunicante posterior en donde asienta la mayoría de los aneurismas que ocasionan una P3N (fig. 1). Las fibras pupilomotoras, destinadas al esfínter pupilar, se localizan en la parte superomedial del motor ocular común, siendo las más vulnerables en casos de compresión del nervio.

Las parálisis agudas del III nervio son debidas casi siempre a isquemia periférica o a compresión por un AAcP, y en el diagnóstico diferencial se ha dado especial relevancia al tamaño pupilar. Así pues, una $\mathrm{P} 3 \mathrm{~N}$ con midriasis total indicaría una compresión, posiblemente por un aneurisma, mientras que una $\mathrm{P} 3 \mathrm{~N}$ con pupila normal sería indicativa de trastorno microvascular. Las P3N con afectación pupilar parcial y anisocoria superior a $0,5 \mathrm{~mm}$ son producidas con cierta frecuencia por lesiones compresivas diferentes de los aneurismas, a nivel cerebral, del seno cavernoso o de la fosa posterior $(1,2)$. Asimismo, si la pupila no está dilatada en un caso de parálisis total de los músculos extrínsecos dependientes del motor ocular común, podemos hablar de preservación pupilar, cosa que no es tan

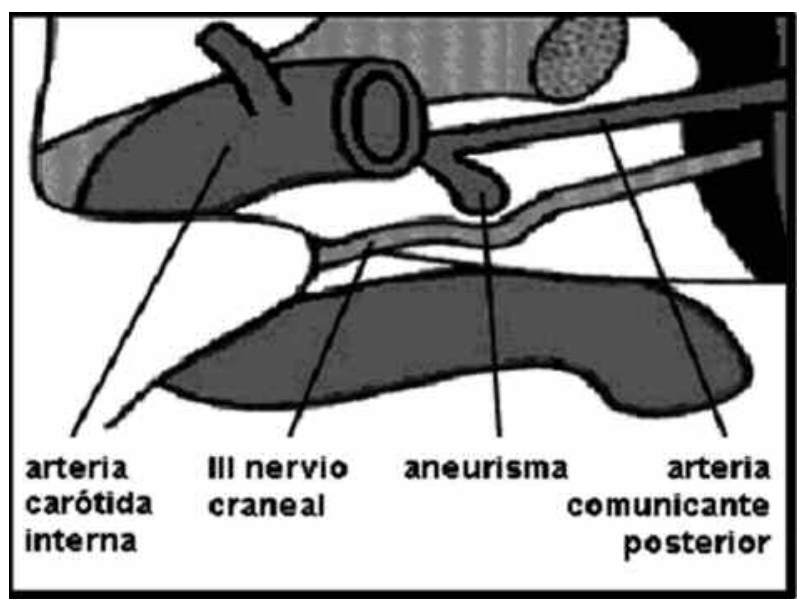

Fig. 1: Relación entre el III nervio craneal y un aneurisma situado en la unión de la arteria comunicante posterior con la arteria carótida interna.

\footnotetext{
${ }^{1}$ Licenciado en Medicina. Servicio de Oftalmología. Hospital Universitari de Bellvitge. Institut Català de Retina. Barcelona. España.

2 Doctor en Medicina. Servicio de Oftalmología. Hospital Universitari de Bellvitge. Institut Català de Retina. Barcelona. España. E-mail: jarruga@csub.scs.es

${ }^{3}$ Licenciado en Medicina. Servicio de Oftalmología. Hospital Universitari de Bellvitge. Barcelona. España.

${ }^{4}$ Licenciado en Medicina. Servicio de Radiología. Hospital Universitari de Bellvitge. Barcelona. España.

5 Doctora en Medicina. Servicio de Radiología. Hospital Universitari de Bellvitge. Barcelona. España.
} 
cierta cuando la afectación motora extrínseca es parcial. Las P3N consecutivas a AAcP se suelen acompañar de dolor orbitario o frontal ipsilateral, por afectación de las fibras trigeminales del III nervio. No obstante, las $\mathrm{P} 3 \mathrm{~N}$ de causa isquémica también pueden cursar con dolor y, a su vez, la ausencia de éste no excluye la causa aneurismática, por lo que la importancia del dolor es relativa para orientar hacia una u otra etiología. En cuanto a la edad del paciente, los aneurismas son excepcionales en menores de 10 años, mientras que en edades avanzadas las $\mathrm{P} 3 \mathrm{~N}$ son en su inmensa mayoría de causa isquémica, sobre todo cuando existen factores de riesgo vascular asociados (diabetes, hipertensión arterial, dislipemia, tabaquismo).

Desde un punto de vista neurorradiológico, la arteriografía por cateterismo (AG) es la prueba «Gold Standard» para la detección de aneurismas intracraneales (fig. 2), aunque se asocia a un riesgo del $1-2 \%$ de complicaciones permanentes, tales como accidentes vasculares cerebrales. Debido a ello, está siendo progresivamente reemplazada por las técnicas de neuroimagen incruentas, como la angiografía por resonancia magnética nuclear (ARM) (3) y la angiografía por tomografía computarizada (ATC) $(4,5)$, cuya incidencia de complicaciones es menor. La ARM no utiliza radiación ionizante ni requiere obligatoriamente el empleo de contraste, por lo que es fácilmente realizable en pacientes con ateromatosis severa o insuficiencia renal. La ATC es un procedimiento rápido y poco costoso que comporta la inyección del contraste por

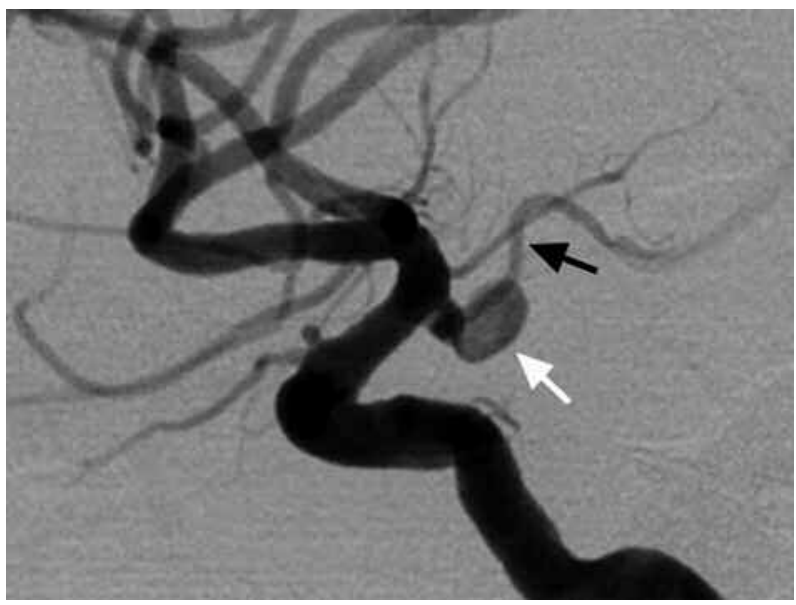

Fig. 2: Arteriografía convencional que muestra un $A A c P$ (flecha blanca) en el origen de la comunicante posterior (flecha negra). vía intravenosa y no en la arteria femoral, a diferencia de la AG. Por su parte, la resonancia magnética nuclear (RMN) simple permite visualizar aneurismas trombosados, a veces no detectables incluso mediante la AG. El tamaño de los AAcP es muy importante, ya que los pequeños (menores de 3-4 $\mathrm{mm}$ ) raramente producen sintomatología clínica, pero los de diámetro inferior a 4-5 $\mathrm{mm}$ son difíciles de detectar con las técnicas de neuroimagen incruentas. El tamaño crítico de un aneurisma causante de una P3N está, pues, alrededor de los $4 \mathrm{~mm}$ $(1,3)$. En este estrecho margen, el AAcP puede ser sintomático, con buena visualización o no, mediante la ARM y la ATC. Entre las dos técnicas citadas, los estudios comparativos han demostrado una mayor sensibilidad de la ATC que de la ARM para la detección de los aneurismas (4) (fig. 3).

En la práctica clínica diaria, los problemas diagnósticos deben ser resueltos a corto plazo, ya que un retraso en el tratamiento puede acarrear graves consecuencias vitales para el paciente. Antes de disponer de las técnicas neurorradiológicas incruentas, se indicaba muchas veces una AG en P3N con afectación pupilar parcial o en parálisis extrínsecas sin afectación pupilar que no mejoraban. Durante los últimos años, los protocolos de actuación han variado considerablemente, reservando la AG para casos seleccionados. En nuestro algoritmo de actuación frente a una P3N aguda aislada (tabla I), hacemos una distinción por edades, de manera que a los pacientes menores de 10 años se les realiza sólo una

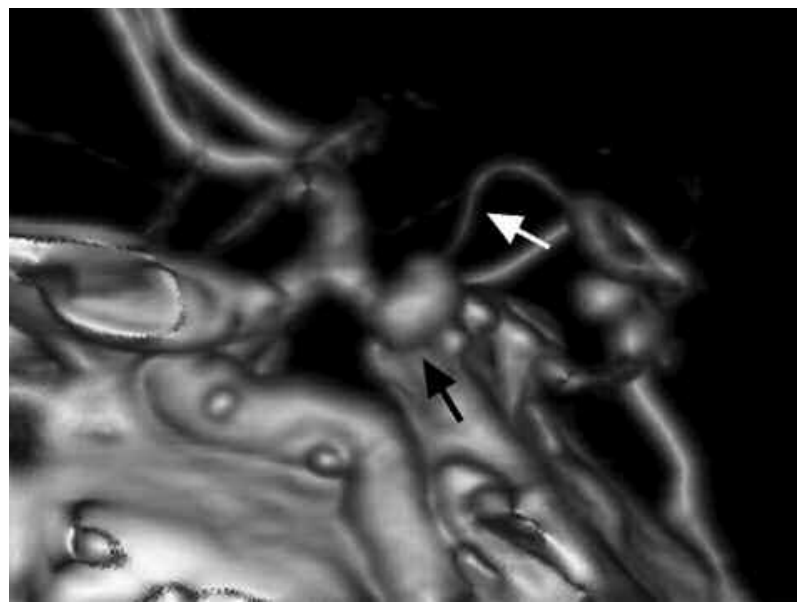

Fig. 3: Angiografía por tomografía computarizada, con reconstrucción $3 D$ del caso anterior, en donde se visualizan el aneurisma (flecha negra) y la mencionada arteria (flecha blanca). 
Tabla I. Protocolo del Hospital Universitari de Bellvitge

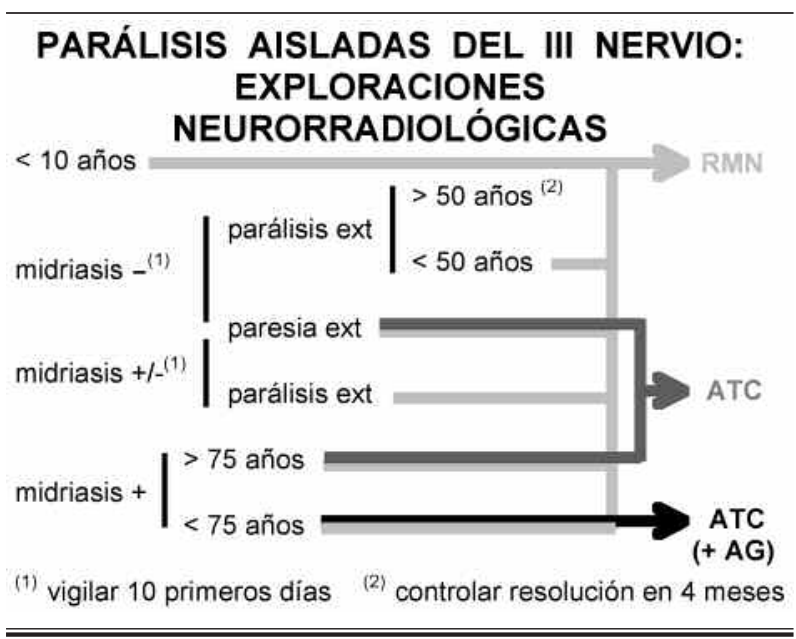

RMN. A partir de esta edad, ante una P3N con midriasis total realizamos además una ATC en primera instancia y, si el resultado es normal, una AG en segunda instancia, teniendo en cuenta el riesgo de AAcP. Por encima de los 75 años sólo efectuamos AG en aquellos casos en los que contemplamos la indicación de un tratamiento endovascular del aneurisma. Cuando la midriasis no es total, pero la paresia extrínseca tampoco lo es, practicamos una RMN, y una ATC ante la remota posibilidad de AAcP. A su vez, en una P3N con afectación pupilar parcial y extrínseca total nos limitamos a la RMN, que nos permitirá descartar las posibles causas tumorales. Por último, si la parálisis total extrínse- ca se acompaña de una pupila normal, sólo indicamos dicha prueba en pacientes menores de 50 años, y proponemos la simple observación a edades más avanzadas. En cualquier caso, ante una P3N con afectación pupilar parcial o sin ella, se debe vigilar siempre estrechamente al paciente durante los primeros 10 días para descartar una progresión hacia la midriasis total. Además, en los casos en que se adopta una conducta conservadora por la sospecha de una afección isquémica microvascular, ésta quedará confirmada sólo si la motilidad ocular se normaliza en el espacio de 4 meses.

\section{BIBLIOGRAFÍA}

1. Lee AG, Hayman LA, Brazis PW. The evaluation of isolated third nerve palsy revisited: an update on the evolving role of magnetic resonance, computed tomography, and catheter angiography. Surv Ophthalmol 2002; 47: 137157.

2. Jacobson DN. Relative pupil-sparing third nerve palsy: etiology and clinical variables predictive of a mass. Neurology 2001; 56: 797-798.

3. Kupersmith MJ, Heller G,Cox TA. Magnetic resonance angiography and clinical evaluation of third nerve palsies and posterior communicating artery aneurysms. J Neurosurg 2006; 105: 228-234.

4. Vaphiades MS, Cure J, Kline L. Management of intracranial aneurysm causing a third nerve palsy: MRA, CTA or DSA. Semin Ophthalmol 2008; 23: 143-150.

5. Mathew MRK, Teasdale E, McFadzean RM. Multidetector computed tomographic angiography in isolated third nerve palsy. Ophthalmology 2008; 115: 1411-1415. 